\title{
THE CLOSED IDEALS OF SOME DIRICHLET AND HYPO-DIRICHLET ALGEBRAS
}

\author{
ROBERT G. BLUMENTHAL
}

\begin{abstract}
We characterize the closed ideals of (i) the Dirichlet algebras discovered by $\mathbf{A}$. Browder and $\mathbf{J}$. Wermer and (ii) the hypoDirichlet algebras discovered recently by A. G. Brandstein. Our results show that the class of closed ideals of each of these algebras is surprisingly restricted.
\end{abstract}

We consider algebras of functions defined by means of singular maps. A homeomorphism $\psi$ of one circle onto another of radius $r$ is called singular if $\psi$ maps a Borel set of Lebesgue measure zero onto a set of measure $2 \pi r$. If $K$ is a compact subset of $C$ with boundary $\partial K$, we denote by $A_{K}$ the subalgebra of $C(\partial K)$ of all functions which admit continuous extensions to $K$, analytic on the interior of $K$.

Let $\Delta=\{z \in C:|z| \leqq 1\}$, the closed unit disc, $T=\partial \Delta$, and $A_{0}=A_{\Delta}$. Let $q$ be a singular homeomorphism of $T$ onto itself. Define $A_{0}(q)=\left\{f \in A_{0}\right.$ : $\left.f \circ q \in A_{0}\right\}$. Then $A$ is a Dirichlet algebra (i.e. $\mathrm{Cl}\left[\operatorname{Re} A_{0}(q)\right]=C_{R}(T)$ ) [3], and a maximal closed subalgebra of $A_{0}$ [1].

THEOREM 1. I is a closed ideal of $A_{0}(q)$ if and only if there exist closed ideals $I_{1}$ and $I_{2}$ of $A_{0}$ such that $I=\left\{f \in I_{1}: f \circ q \in I_{2}\right\}$.

Suppose now that $q \circ q=$ identity. Let $T / q$ denote the quotient space of $T$ induced by $q$. Set $A_{q}=\left\{f \in A_{0}: f \circ q=f\right\}$. The subalgebra of $C(T / q)$ induced by $A_{q}$ is a Dirichlet algebra on $T / q$, as well as a maximal closed subalgebra of $C(T / q)[3]$.

THEOREM 2. I is a closed ideal of $A_{q}$ if and only if there exists a closed ideal $J$ of $A_{0}$ such that $I=A_{q} \cap J$.

Let $\Gamma$ be the annulus $\{z \in C: 1 \leqq|z| \leqq 2\}$, let $T^{\prime}=\{z \in C:|z|=2\}$, and let $B_{0}=A_{\Gamma}$. Let $p$ be a singular homeomorphism of $T$ onto $T^{\prime}$ which is orientation preserving. Define $B_{p}=\left\{f \in B_{0}: f(z)=f(p(z))\right.$, all $z$ in $\left.T\right\}$. The restriction of $B_{p}$ to $T$ is a hypo-Dirichlet algebra (i.e. there exist $f_{1}, \cdots, f_{n}$ in $B_{p}$ whose analytic extensions to $\Gamma$ are never zero such that the real vector space spanned by $\operatorname{Re} B_{p}$ and $\log \left|f_{1}\right|, \cdots, \log \left|f_{n}\right|$ is dense in $C_{R}(T)$ ) [2].

Received by the editors June 16, 1971.

AMS 1970 subject classifications. Primary 46J10, 46J20.

Key words and phrases. Singular homeomorphism, analytic function, closed ideal, dual space of $C(X)$.

(c) American Mathematical Society 1972 
THEOREM 3. I is a closed ideal of $B_{p}$ if and only if there exists a closed ideal $J$ of $B_{0}$ such that $I=B_{p} \cap J$.

We identify the dual space $C(X)^{*}$ of $C(X), X$ compact, with the space of all complex, regular, Borel measures on $X$. If $A$ is a subspace of $C(X), A^{\perp}$ is the space of all $\mu \in C(X)^{*}$ such that $\int f d \mu=0$ for each $f \in A$.

If $A$ is a closed subalgebra of $C(X)$ and $I$ is a closed ideal of a subalgebra of $A$, we let $I[A]$ denote the closed ideal of $A$ generated by the functions in $I$.

LEMMA 1. Let $A$ and $B$ be closed subalgebras containing 1 of $C(X)$. Suppose there exists a Borel subset $E$ of $X$ such that $|v|(E)=|\mu|(X-E)=0$ for every $\mu \in A^{\perp}, \nu \in B^{\perp}$. If $I_{1}$ and $I_{2}$ are closed ideals of $A$ and $B$ respectively, then $I_{1} \cap I_{2}$ is a closed ideal of $A \cap B$, and all the closed ideals of $A \cap B$ arise in this way.

Proof. Let $I$ be a closed ideal of $A \cap B$. We shall show that $I=$ $I[A] \cap I[B]$. Clearly $I \subseteq I[A] \cap I[B]$. To prove the reverse containment we shall prove $I^{\perp} \subseteq(I[A] \cap I[B])^{\perp}$. Thus, let $\eta \in I^{\perp}$ and $f \in I[A] \cap I[B]$. We have to show $\int f d \eta=0$.

Since $I[A]=\mathrm{Cl}(A \cdot I)$ and $I[B]=\mathrm{Cl}(B \cdot I)$, there exist sequences $\left\{g_{n}\right\}$ and $\left\{h_{n}\right\}, g_{n} \in A \cdot I, h_{n} \in B \cdot I$, which converge uniformly to $f$. Since

$$
\int f d \eta=\operatorname{Lim}_{n \rightarrow \infty} \int_{E} g_{n} d \eta+\operatorname{Lim}_{n \rightarrow \infty} \int_{X-E} h_{n} d \eta
$$

it suffices to show $\int_{E} g_{n} d \eta$ and $\int_{X-E} h_{n} d \eta$ are zero for each $n$.

Since $g_{n} \in A \cdot I, g_{n}=\sum_{r=1}^{p} a_{r} i_{r}$, where $a_{r} \in A$, and $i_{r} \in I$. Observe that $i_{r} \eta \in(A \cap B)^{\perp}$. It is known that $(A \cap B)^{\perp}=A^{\perp}+B^{\perp}$ [3, p. 547]; hence $i_{r} \eta=\mu_{r}+v_{r}, \mu_{r} \in A^{\perp}, v_{r} \in B^{\perp}$. Then

$$
\int_{E} g_{n} d \eta=\sum_{r=1}^{p}\left(\int_{E} a_{r} d \mu_{r}+\int_{E} a_{r} d \nu_{r}\right)=\sum_{r=1}^{p} \int a_{r} d \mu_{r}=0 .
$$

Similarly $\int_{X-E} h_{n} d \eta=0$. Q.E.D.

Proof OF THEOREM 1. If we set $A_{0}^{q}=A_{0} \circ q^{-1}=\left\{f_{\circ} q^{-1}: f \in A_{0}\right\}$, then $A_{0}(q)=A_{0} \cap A_{0}^{q}$ where, as observed in [3], $A_{0}$ and $A_{0}^{q}$ satisfy the hypothesis of Lemma 1. Since every closed ideal of $A_{0}^{q}$ has the form $I_{2} \circ q^{-1}$, where $I_{2}$ is a closed ideal of $A_{0}$, Theorem 1 follows.

Suppose next that $A$ is a closed subalgebra of $C(X)$ containing 1 and that $G$ is a group of homeomorphisms of $X$ onto itself, such that $f \circ g \in A$ for every $f \in A, g \in G$. For $S \subseteq C(X)$ set $S_{G}=\{f \in S: f \circ g=f$ for every $g \in G\}$.

LEMma 2. Assume $G$ is finite. Then if $J$ is a closed ideal of $A, J_{G}$ is a closed ideal of $A_{G}$ and every closed ideal of $A_{G}$ arises in this way. 
Proof. Let $I$ be a closed ideal of $A_{G}$. We will prove that

$$
I=[\mathrm{Cl}(A \cdot I)]_{G} \text {. }
$$

We show first

$$
[\mathrm{Cl}(A \cdot I)]_{G}=\mathrm{Cl}\left[(A \cdot I)_{G}\right]
$$

For suppose that $f \in[\mathrm{Cl}(A \cdot I)]_{G}$. Then there exists a sequence $\left\{f_{n}\right\}$, $f_{n} \in A \cdot I$, which converges uniformly to $f$. Let $N$ be equal to the order of $G$. Then, if $F_{n}=N^{-1} \sum_{g \in G} f_{n} \circ g, F_{n} \in(A \cdot I)_{G}$ and $F_{n} \rightarrow N^{-1} \sum_{g \in G} f \circ g=f$. Hence $f \in \mathrm{Cl}\left[(A \cdot I)_{G}\right]$, proving (2).

Since $I \subseteq[\mathrm{Cl}(A \cdot I)]_{G}$, in order to prove (1) it suffices to prove that $I^{\perp} \subseteq[\mathrm{Cl}(A \cdot I)]_{G}^{\perp}$. Thus if $\eta \in I^{\perp}$, we need only show (by (2)), that $\eta$ annihilates $(A \cdot I)_{G}$. Let $h \in(A \cdot I)_{G}$, where $h=\sum_{r=1}^{p} a_{r} i_{r}, a_{r} \in A, i_{r} \in I$. Since $i_{r} \eta \in A_{G}^{\frac{1}{G}}$ we have

$$
\begin{aligned}
\int h d \eta & =\frac{1}{N} \int\left(\sum_{g \in G} h \circ g\right) d \eta=\frac{1}{N} \int \sum_{g \in G} \sum_{r=1}^{p}\left(a_{r} \circ g\right) \cdot i_{r} d \eta \\
& =\frac{1}{N} \sum_{r=1}^{p} \int\left(\sum_{g \in G} a_{r} \circ g\right) \cdot i_{r} d \eta=0 .
\end{aligned}
$$

Proof of Theorem 2. Let $G^{\prime}$ be the two element group of homeomorphisms of $T$ generated by $q$ (so that $\left.A_{q}=\left(A_{0}\right)_{G^{\prime}}\right)$. If $I$ is a closed ideal of $A_{q}$, we obtain by means of Lemma 2 and Theorem 1

$$
\begin{aligned}
I & =\left(I\left[A_{0}(q)\right]\right)_{G^{\prime}}=\left(I\left[A_{0}\right] \cap I\left[A_{0}^{q}\right]\right)_{G^{\prime}}=\left(I\left[A_{0}\right]\right)_{G^{\prime}} \cap\left(I\left[A_{0}^{q}\right]\right)_{G^{\prime}} \\
& =\left(I\left[A_{0}\right]\right)_{G^{\prime}}=A_{q} \cap I\left[A_{0}\right] .
\end{aligned}
$$

Proof of TheOREM 3. Define $p_{1}: T \cup T^{\prime} \rightarrow T \cup T^{\prime}$ by $p_{1}\left|T=p, p_{1}\right| T^{\prime}=$ $p^{-1}$. Then $p_{1} \circ p_{1}=$ identity, $T \cup T^{\prime} / p_{1}$ may be identified with $T$, and if $G^{\prime \prime}$ denotes the two element group of homeomorphisms of $T \cup T^{\prime}$ on itself generated by $p_{1}, B_{p}=B_{G^{m}}$. Thus the proof of Theorem 3 is analogous to the proof of Theorem 2. Q.E.D.

\section{BIBLIOGRAPHY}

1. R. G. Blumenthal, Maximality in function algebras, Canad. J. Math. 22 (1970), 1002-1004.

2. A. G. Brandstein, A class of hypo-Dirichlet algebras, Proc. Amer. Math. Soc. 28 (1971), 501-504.

3. A. Browder and J. Wermer, A method for constructing Dirichlet algebras, Proc. Amer. Math. Soc. 15 (1964), 546-552. MR 29 \#2669.

Department of Mathematics, University of Miami, Coral Gables, Florida 33124

Current address: Department of Mathematics, Wellesley College, Wellesley Massachusetts 02181 